\title{
Study on Adaptive Learning System based on Network
}

\author{
An Wang ${ }^{1,}$ a, Miaomiao $\mathrm{Li}^{2, \mathrm{~b}}$ \\ ${ }^{1}$ Education School, Jiangxi Science \& Technology Normal University, Nanchang, China \\ ${ }^{2}$ Jiangxi Science \& Technology Normal University, Nanchang, China \\ awanganwangmenghua@163.com, blimiaom8899@163.com
}

Keywords: adaptive learning system; network education.

\begin{abstract}
With the continuous development of network education in China, how to develop a web-based and learner-centered on-line adaptive learning system has become a hotspot in the research of network education and learning platform, which can stimulate the greatest interest of learners to realize the real autonomous learning. The paper mainly discusses the meaning and characteristics of the network adaptive learning, and analyzes its architecture.
\end{abstract}

\section{Introduction}

In the era of knowledge explosive growth, the starting point and foothold of national education reform is on the cultivating students' quality and improving students' ability. Because of uneven learning base, they are different from the learning method, ability, progress and efficiency, which determine different students are distinguished in teaching process, teaching methods and teaching contents. Traditional classroom teaching gradually can not meet the rapid development of modern education due to time and space constraints. But distance education based on web adopts asynchronous interactive learning mode, and has advantages of free time and space, open system, and convenient cooperation, which break the constraints of time and space, meet the demand of multimedia learning, and provide open and autonomous virtual learning environment for students.

\section{The meaning of adaptive learning under the network environment}

Adaptive learning based on network refers to a learning system that, under the network environment, learners can formulate their own learning plan according to their actual situation and choose suitable learning content and learning method, so as to make progress.

Adaptive learning based on network has the following characteristics. First, Adaptive learning based on network is independent learning centered on the learners. Autonomous learning, actually the learning of metacognitive monitoring, is the process in which the learners actively choose learning content, improve learning method, implement the learning process and adjust the degree of effort according to their learning needs, learning ability and learning goals. Autonomous learning has the characteristics of the autonomy, initiative, effective and independent. It fully reflects subjective status of learners.

Secondly, the adaptive learning under the network environment is individualized learning. The adaptive learning under the network environment fully embodies a new concept system embodied in individualized learning, including learning centered, learning for all learners, for learners to learn everything, everything for learners and other service concept. The adaptive learning under the network environment lets the network learning content adapted to each individual learner and the basic requirement of realizing individual learning. The learning content under the network environment will use the structural mode and various expression mode that learners can easily accept, which requires learners to have the independent consciousness and join into study group to make themselves have independent learning ability. From this sense, the adaptive learning under the network environment is an individual learning. 
Third, the adaptive learning under the network environment is personalized learning. The learners are the individuals with their own unique personality. In the adaptive learning under the network environment, the learners can consider the learning environment and social demand to choose and modify the learning direction and target, and to arrange learning activities sequence, time step, learning methods and so on according to their own needs, abilities, interests and psychological characteristics, so as to make the learners' personality fully reflected and realize personalized learning.

Fourth, the adaptive learning under the network environment is self-monitoring learning. In essence, it is the learning of learners' self awareness, self control and self regulation. Self monitoring is the basic prerequisite and basic guarantee of self development and realization in the adaptive learning under the network environment, which is achieved through the feedback mechanism. In the whole process of adaptive learning under the network environment, learners autonomously adjust and determine the learning objectives and objectives, select the appropriate strategies of solving problems, monitor the learning process, and acquire feedback continuously according to self need, behavior effect and external reaction, so as to get the optimal personalized development through positive self monitoring behavior.

\section{The necessity of realizing the adaptive learning based on network}

Rich learning resources. In the adaptive learning based on network, in order to freely choose their own learning goals and styles, the learning resources must be rich, which is the basic condition of carrying out the adaptive learning based on network. We can say that the adaptive learning based on network is a kind of learning based on resources and it can fit different needs of various learners.

Timely and accurate information feedback. In the adaptive learning based on network, learners mainly cultivate their self awareness through self monitoring and regulation of cognitive activities, and get timely feedback information in the process of monitoring the learning. With the feedback, learners can discover the problems in the process of cognitive activities, and then adjust their learning strategies, so as to reduce the blindness in the learning process, which can improve the efficiency of cognitive activities and the probability of success. If lacking information feedback in network education, the real adaptive learning is unable to be carried out.

Objective and accurate evaluation. Objective and accurate assessment is very important to learners. The main function of evaluation is diagnosis and regulation. Specifically, that is, learners diagnose their learning through feedback, timely find out problems in the learning and then adjust the learning process, so as to achieve their learning objectives. In the process of adaptive learning based on network, learners should carry out accurate and objective evaluation according to information of many aspects. They can grasp their learning situation and control the learning process. That can be said that, learning activity is a kind of evaluation activity, because it is an important basis for learners to understand their own learning ability.

\section{The architecture of adaptive learning system based on network}

The part of learning. The learning part embodies the basic characteristic of the adaptive learning based on network. When learners want to learn some resources in the internet, they need to register account first, and then enter into the adaptive learning system according to this account. If the learners are willing to, the system will assess their knowledge level. Because of great difference in knowledge level and ability, learners need to determine the appropriate learning objectives and strategies according to their own situation under certain guidance. Learner can see whether they achieve the setting goals through adaptive testing, which can be carried out step by step in the process of learning whenever according to learning content. Of course, learner can also communicate with other learners, gradually get feedback, and then adjust learning process once formulated, to meet the learners' need of personalized learning. 
The part of teaching. The logical rules made in teaching part are mainly to meet the specific needs of learners. In the adaptive learning system based on network, the instructor, as the leader, can interact with any part of the system, but, if the instructor wants to communicate with the learner, the learner must have this demand; otherwise it would run counter to one's desire and affect the learning. The main task of teaching part is: when the learner has the demand, the instructor helps the learners to do some assessment on the learning situation, and then guides them to determine the learning plan and strategy according to the assessment. In the adaptive learning system, all the instructors do are made for guiding the learners and then make them better realize personalized adaptive learning.

Supporting part. The supporting part is mainly composed of adaptive test question database, learner record library, and knowledge base composition. The content of each database can be rewritten by the system according to the certain principle, and also can be edited by the guidance in the scope of authority. In the adaptive learning system based on network, the supporting part is the fundamental guarantee for learners to realize adaptive learning.

The part of management and maintenance. The main work of the management and maintenance part is establishing the test question bank, dividing teaching content into interrelated and relatively independent cognitive unit, and teaching those cognitive elements. The teaching is carrying out in different way. When learners encounter problems in learning cyber source, the main function of management and maintenance is to coordinate the collaboration between learners. The other function of the management and maintenance is to investigate learners' knowledge level $n$ the process of learning, and then propose the corresponding suggestions according to the specific situation.

\section{Summary}

With the development of intelligent network learning technology, the adaptive learning system can provide relative good network learning environment for learners, and constantly improve their learning ability, so as to better promote the realization of the real adaptive learning based on network.

\section{References}

[1]ChenTY, Zhang JP. An agent-based adaptive learning system (AB-ALS) [A]. Proceedings of the First InternationalCon.f on Innovative Computing, Information and Control [C]. 2006: 378 -381.

[2]Kamel N N, Davsion R M.Applying CSCW Technology to Overcome Traditional Barriers in Group Interactions[J]. Information\&Management, 1998,34: 209-219.

[3] Hetherington RG, Sood S, AdvaniB. A prototype distributed adaptive learning system [A]. Proceedings of the International Conference on Computers in Education (ICCE.02) [C]. 2002: 576 $-577$.

[4] LiX, Xie Z, Qiu Y H. Adaptivity implementation based on multi -a-gent technology [A]. Proceeding of the IEEE International Con.f on Advanced Learning Technologies [C]. 2004: 146 -150 .

[5]WeberG, BrusilovskyP. ELM -ART: an adaptive versatile system for Web -based instruction [J]. International Journal of Artificial Intelligence in Education, 2001: 351 -384.

[6]Baoguo He, Guangshe Zhao, Meilan Liu. Research on adaptive learning system in the network environment[J]. Computer measurement and control, 2008,16(2):256-258,284.

[7]Xiaohong Gao. Research on adaptive learning system based on network[J]. College of mathematical and physical sciences of shanghai normal university, 2003. 